\title{
The Interaction of Imposed and Inherent Olfactory Mucosal Activity Patterns and their Composite Representation in a Mammalian Species Using Voltage-Sensitive Dyes
}

\author{
Paul F. Kent, ${ }^{1,3}$ Maxwell M. Mozell, ${ }^{1,2}$ Stephen J. Murphy, ${ }^{1,2}$ and David E. Hornung ${ }^{1,2}$ \\ 'Olfactory Research Center, Departments of ${ }^{2}$ Physiology and ${ }^{3}$ Neurology, SUNY Health Science Center, \\ Syracuse, New York 13210
}

From amphibian data, two mechanisms that could underlie the encoding of odorants by the mucosal activity patterns they engender are as follows: (1) receptors with similar odorant selectivities could be aggregated spatially on the mucosa (inherent patterns); (2) in analogy to gas chromatography, as odorants are drawn along the surface of the mucosa the strongly sorbed ones could be deposited preferentially upstream, whereas the weakly sorbed ones could be distributed more evenly (imposed patterns). Do both of these possible coding mechanisms operate in mammals and, if so, how do they interact in giving composite patterns (imposed + inherent)? Fluorescence changes in dl-4-ANEPPS applied to rat mucosas were monitored by a $10 \times 10$ pixel photodiode array. To observe the inherent patterns, three odorants of varying sorbabilities first were puffed uniformly onto the entire mucosa mounted in a Delrin chamber. To bring out the imposed patterns, the chamber was then sealed to replicate anatomically the rat's nasal cavity, and these same odorants were drawn at three flow rates along the mucosal flow path. The results demonstrated for the first time the existence of imposed patterns in a mammal. The strongly sorbed odorants, unlike the weakly sorbed one, showed marked imposed patterns. Within physiological limits, increasing the flow rate decreased the magnitude of the imposed patterns. One might consider strategies that the olfactory process could use either to negate or to take advantage of the chromatographic effect, because the lability of the composite patterns with changing stimulus conditions raises questions about their role in odorant encoding.

Key words: olfaction; mucosal activity patterns; voltage-sensitive dyes; optical recording; rat; odorant encoding; olfactory mucosa
There is ample evidence from amphibia (Kauer and Moulton, 1974; Moulton, 1976; Kubie et al., 1980; MacKay-Sim and Kubie, 1981; MacKay-Sim et al., 1982; Cinelli et al., 1995) and rats (Thommesen and D'Oving, 1977; MacKay-Sim and Kesteven, 1994; Ezeh et al., 1995) that different odorants produce different reproducible spatial patterns of activity with differing regions of greater and lesser response magnitudes across the mucosal surface. Most of these investigators puffed the odorant directly down onto the exposed olfactory mucosa and recorded individual clcctro-olfactograms (EOGs) in successive order from different mucosal positions. Responses to repeated presentations of the same and different odorants, often in different animals, were then combined into EOG spatial collages. These differential odorant spatial activity patterns also have been shown using voltagesensitive dyes (Kent and Mozell, 1992; Youngentob and Kent, 1995; Youngentob et al., 1995) which, unlike the EOG technique, record the activity from a large number of mucosal locations simultaneously, thus greatly increasing the resolution of the activity patterns and greatly reducing any confounding effects caused by repetitive stimulations over long periods of time. These differential regional activity patterns were termed "inherent" activity patterns by Moulton (1976), implying that they are caused by

\footnotetext{
Received May 17, 1995; revised Aug. 28, 1995; accepted Sept. 5, 1995.

This work was supported by National Institutes of Health Grant DC00072.

Correspondence should be addressed to Dr. Paul F. Kent, Department of Physiology, Room 3225 Weiskotten Hall, SUNY Health Science Center, 766 Irving Avenue, Syracuse, NY 13210-2339.

Copyright (C 1995 Society for Neuroscience $0270-6474 / 95 / 160345-09 \$ 05,00 / 0$
}

receptors that, having similar inherent odorant sensitivities, are aggregated inherently into the same mucosal regions.

On the other hand, when the odorants, rather than being puffed down, are drawn instead through the olfactory sac (approximating their normal flow along the mucosal surface), another type of activity pattern is observed. That is, different odorants produce differently sloped gradients of activity from the beginning to the end of the flow path (Mozell, 1966, 1970). In a series of electrophysiological (Mozell, 1966, 1970; Mozell et al., 1984, 1987), radioisotope (Hornung and Mozell, 1977; Ilornung et al., 1981), and gas chromatographic (Mozell and Jagodowicz, 1973, 1974) studies, evidence has been presented showing that these differential response gradients are attributable to a process analogous to gas chromatography whereby the greater an odorant's sorption to the mucosa, the greater the percentage of its molecules sorbed at the beginning of the flow path and the fewer the number arriving downstream. Moulton called these activity patterns "imposed," highlighting that they are not attributable to inherent biological factors but rather to physicochemical principles that impose different mucosal distributions for the molecules of different odors.

Because mucosal activity patterns have been proposed as underlying olfactory discrimination, the question arises as to how these two types of activity patterns, and the mechanisms thought to underlie them, interact. Note that although inherent patterns can be produced more or less uncomplicated with imposed patterns by puffing the odorants dircetly down onto the mucosa, the reverse is not true. That is, in drawing the odorants across the mucosa to set up the molecular gradients basic to imposed patterns, these gradients necessarily fall across regions of differing 


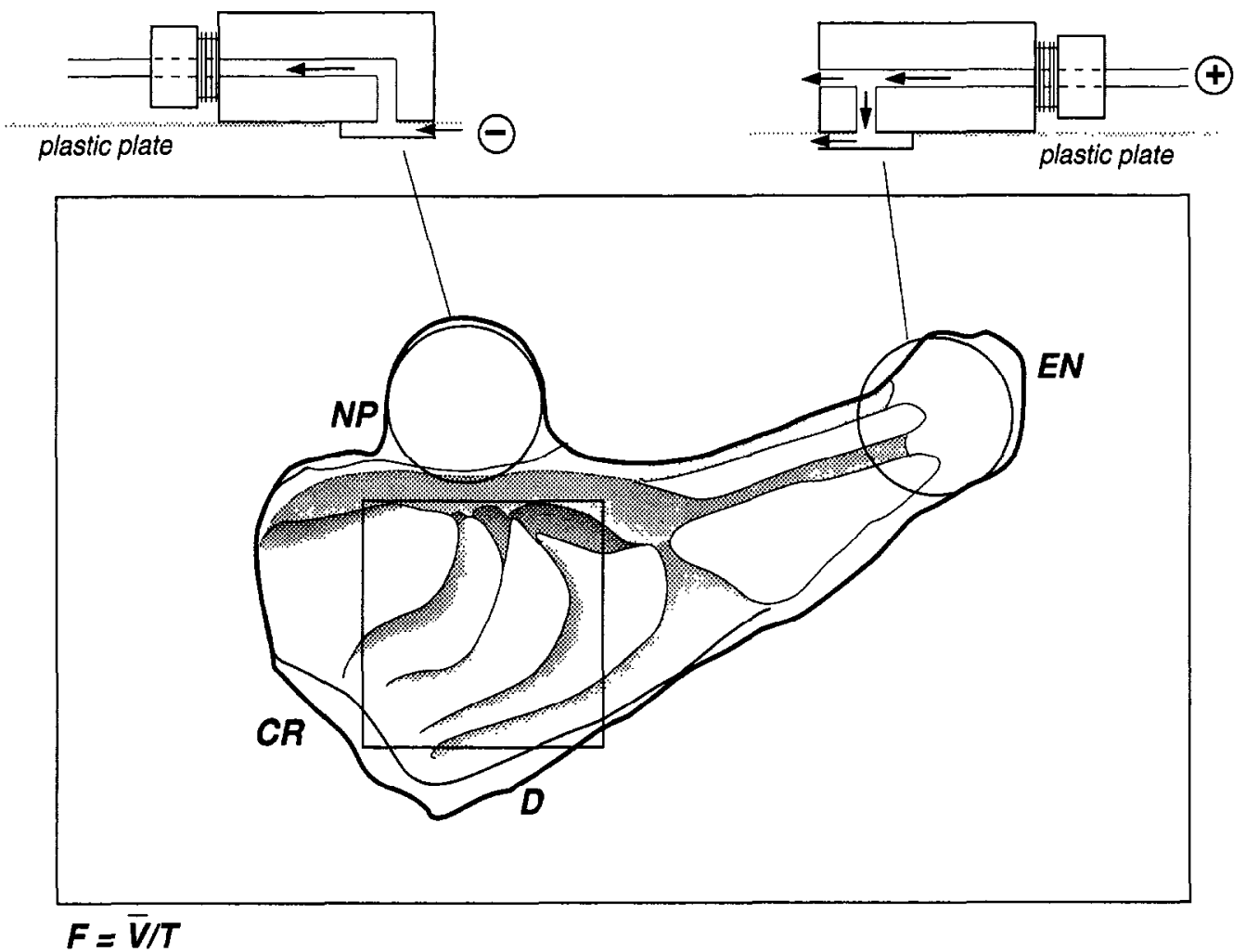

Figure 1. Schematic drawing of the chamber containing the mucosa lining the medial surface of the turbinates and the apparatus used to produce a more natural sniff. The square indicates the area from which the photodiodes record. The insets show the Delrin plugs fitted through the plastic plate into the external nares and the nasopharynx allowing the constant flow of either deodorized or odorized air across the mucosal lining. The arrows in the Delrin plugs show the flow of air. For the nasopharynx, the flow through the Delrin plug is drawn at different flow rates by negative pressure. For the external naris plug, a $600 \mathrm{cc} / \mathrm{min}$ flow under positive pressure can either bypass the nasal cavity altogether or be drawn into it by the negative pressure applied to the plug in the nasopharynx. All flows are controlled with mass flow controllers (Teledyne Hastings, Hampton, VA). $E N$, external naris; $N P$, nasopharynx; $D$, dorsal; $C R$, cribriform plate.

selective sensitivities, thus interacting the imposed patterns with the inherent patterns to give "composite patterns." Although these patterns were observed indirectly in an earlier study using frogs (Mozell et al., 1987), the techniques used did not allow the actual visualization of the imposed and inherent patterns or the effect each had on the other, i.e., the composite patterns. It remained, as done here, for the much greater spatial resolution of the voltage-sensitive dye technique to describe the point-by-point appearance of the imposed and inherent activity patterns. The imposed patterns were then extracted from the composite patterns by subtracting the inherent pattcrns from the composite patterns. In recording these patterns, the present study was the first to demonstrate that imposed patterns occur in mammals.

\section{MATERIALS AND METHODS}

The neurophysiological methods summarized below have been described in detail previously (Kent and Mozell, 1992; Youngentob and Kent, 1995; Youngentob et al., 1995).

Mucosal preparation. Eight male Long-Evans hooded rats $(9$ months of age) were housed individually and maintained in a temperature- and humidity-controlled environment. Before surgery the rats were anesthetized by $\mathrm{CO}_{2}$ inhalation and then killed by decapitation. The right nasal cavity was split into halves along its longitudinal axis, exposing the medial turbinates en bloc for neurophysiological evaluation. The excised mucosa was soaked for $20 \mathrm{~min}$ in the fluorescent probe di-4-ANEPPS (Molecular Probes, Eugene, OR) at a concentration of $1 \mathrm{mg} / 100 \mathrm{ml}$ Ringer's solution (in mM: $140.0 \mathrm{NaCl}, 2.0 \mathrm{Na}_{2} \mathrm{HPO}_{4}, 5.6 \mathrm{KCl}, 2.2 \mathrm{CaCl}_{2}, 1.0 \mathrm{MgCl}_{2}, 5.0$ HEPES, 10.0 glucose; $\mathrm{pH}$ adjusted to 7.3 ). After removal from the dye, the tissue was rinsed in Ringer's solution for $20 \mathrm{~min}$ to remove any unbound dye. The preparation was then placed in a small Delrin mounting block and fixed into place using dental cement as shown in Figure 1.

Optics. Using epi-illumination, a real fluorescence image of the object stained with di-4-ANEPPS was projected onto a $10 \times 10$ photodiodc array (Centronics, Newbury Park, CA). Briefly, light from a $24 \mathrm{~V} 250 \mathrm{~W}$ tungsten-halogen lamp was collimated, and the infrared wavelengths were filtered out with a KG-3 Schott heat filter. The light was made quasimonochromatic with a $530 \mathrm{~nm}(65 \mathrm{~nm}$ half-bandwidth) excitation filter and focused onto the preparation with a $50 \mathrm{~mm}$ f1.4 Nikkor lens. The emitted fluorescence wavelengths from the preparation were then transmitted through a dichroic mirror and subsequent $650 \mathrm{~nm}(100 \mathrm{~nm}$ half-handwidth) emission filter. An image was formed at the focal plane of an image lens (38 mm diameter, $125 \mathrm{~mm}$ focal length). With a magnification of $2.5 \times$ and with each photodiode being $1.4 \times 1.4 \mathrm{~mm}^{2}$, each photodiode received light from a $560 \times 560 \mu \mathrm{m}^{2}$ area of the mucosal object. The consistency of alignment between preparations has been described previously (Youngentob and Kent, 1995; Youngentob et al., 1995).

Electronics. In brief, the output of each photodiode was connected to a current-voltage converter (feedback resistance $=33 \mathrm{M} \Omega$ ). After coupling (time constant $=10.3 \mathrm{sec}$ ), the AC signals were amplified by a factor of 1000 , multiplexed, and digitized sequentially ( $12 \mathrm{bits}, 9 \mu \mathrm{sec} / \mathrm{conversion}$ ). This allowed all of the 100 channels to be sampled as quickly as once every millisecond. As described previously (Youngentob et al., 1995), the raw recorded response was corrected for both baseline drift caused by photobleaching and for background fluorescence, which differed from photodiode to photodiode. (See Fig. 2.)

Odorants. The three odorants used in this study [propyl acetate, ethyl acetoacctate, and L-carvone (Aldrich Chemical, Milwaukec, WI)] were chosen because they represent a range of mucosal sorption strengths from 


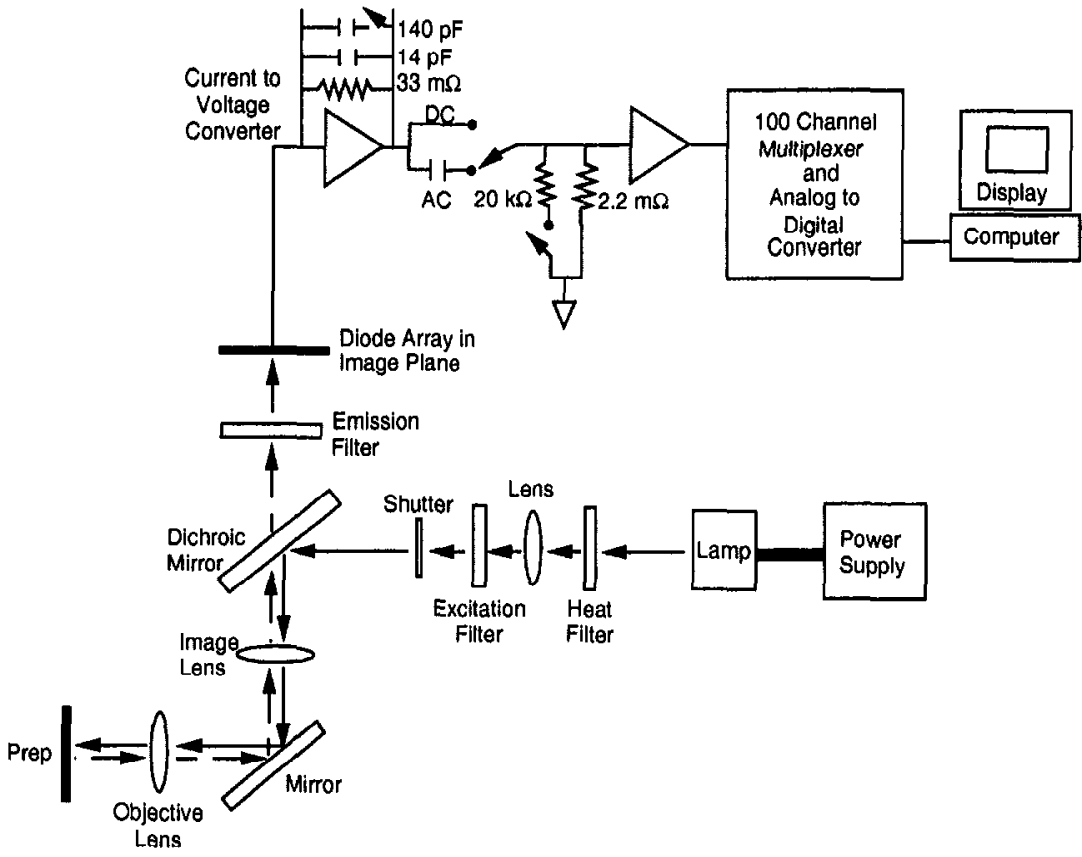

Figure 2. Schematic drawing of the apparatus used to record changes in fluorescence from the mucosa stained with the voltage-sensitive dye from all 100 photodiodes (Kent and Mozell, 1992). See Materials and Methods for details. the weakly sorbed propyl acetate to the strongly sorbed L-carvone. As in earlier studies, a fourth odorant, amyl acetate (Aldrich), was used as a standard stimulus to adjust for any gradual changes in the response size that might occur over time. Amyl acetate was chosen as the standard because it gives a relatively uniform and large response across the mucosa. The concentration (percentage vapor saturation at $23^{\circ} \mathrm{C}$ ) for each odorant was chosen such that it fell within the dynamic range of an odorant's concentration-response function. These concentrations were as follows: $2 \%$ propyl acetate, $30 \%$ ethyl acetoacetate, $30 \%$ L-carvone, and $2 \%$ for the amyl acetate standard. These concentrations were controlled using a previously described flow dilution olfactometer (Mozell, 1970) now modified with mass flow controllers (Youngentob, 1991).

Protocol. Each experimental session consisted of 17 odorant presentations. The first five presentations, which began and ended with the amyl acetate standard, included the three odorant stimuli in random order. As described previously (Kent and Mozell, 1992), each odorant was puffed down uniformly onto the turbinates via a circular Teflon ring $(1 \mathrm{~cm}$ diameter) positioned between the preparation and the optical system in such a way that light could pass to and from the mucosa without interruption. The ring had a number of ports around its circumference to provide the tissue with both a uniform distribution of humidified air between trials and a uniform distribution of odorant molecules during stimulation. All stimuli in this section were presented at a total flow rate of $220 \mathrm{cc} / \mathrm{min}$ for $0.34 \mathrm{sec}$ ( $\mathrm{vol}=1.25 \mathrm{cc}$ ).

After puffing down the first five odorants, a clear plastic plate was placed over the preparation to form a chamber. The smooth surface of the plastic plate replaced the relatively smooth surface of the septum and, with the aid of dental cement, was designed to replicate anatomically the rat's nasal cavity (Fig. 1). Two holes were drilled into the plastic plate. They were positioned to represent the external naris and the nasopharynx so that, when fitted with the Delrin plugs shown in Figure 1, odorized air could be drawn through the nasal cavity across the mucosa lining the turbinates. As with the first five odorant presentations, the volume of the stimulus was $1.25 \mathrm{cc}$, which is in keeping with the dynamic range of the unilateral total inspiratory sniff volumes for a sniffing bout reported by Youngentob et al. (1987). To keep the total number of molecules constant for a given odorant at a given concentration for all flow rates, the required increments in flow rate were achieved by proportional decrements in stimulus presentation time (Mozell et al., 1984). The flow rate of the stimulus was also in the dynamic range of the inspiratory flow rate reported by Youngentob et al. (1987), taking into consideration that only one side of the nose was sampled in the current study. These flow rates were 110,220 , and $440 \mathrm{cc} / \mathrm{min}$, which required $0.68,0.34$, and $0.17 \mathrm{sec}$ presentation times, respectively. Before proceeding, nasal patency and the presence of an air-tight chamber were confirmed by determining whether the exiting airflow was the same as that entering. Under these conditions, the next 12 odorant presentations consisted of drawing each of the three odorants across the mucosa at each of three flow rates in random order. Presentations of the standard amyl acetate stimulus were given at the beginning, midpoint, and end of this series of stimulations.

\section{RESULTS}

As an example of the raw responses developed on the olfactory mucosa, the responses from one rat to one of the three odorants, carvone, are shown in Figure 3. Two of these arrays show the responses when carvone was drawn across the mucosa at the low $(A)$ and high $(B)$ flow rates, both of which gave composite patterns. In the third array $(C)$, carvone was puffed down onto the mucosa giving an inherent pattern. This latter array $(C)$ depicting carvone's inherent pattern shows a pixel-by-pixel distribution of response magnitudes that covers a broad swath across the mucosal sheet. In contrast, when drawn along the mucosal flow path at a low flow rate $(A)$, this highly sorbed odorant produced in this animal a sharp gradient of activity beginning with the most marked responses at the point of entry onto the turbinates followed by progressively smaller responses toward the nasopharynx. However, when the flow rate was increased $(B)$, several differences were observed: (1) the absolute response magnitudes increased pixel-by-pixel over those at the low flow rate; (2) the gradient of activity along the mucosal flow path became greatly muted; and (3) the composite pattern of the relative responses began to resemble the inherent pattern [i.e., $B$ looks more like $C$ than $A$ does]. The raw responses (not shown but represented in the averaged responses given in Fig. 4) to the other strongly sorbed odorant, ethyl acetoacetate, paralleled those of carvone. They also showed a distinct inherent pattern and a composite pattern that, at the low flow rate, displayed a steep gradient along the flow path. As with carvone, increasing the flow rate increased the pixel-by-pixel response magnitudes of the composite pattern and greatly muted the response gradient. This muting resulted in the composite pattern more closely resembling the inherent pattern. As for carvone and ethyl acetoacetate, the raw responses in this animal to the weakly sorbed odorant, propyl acetate, showed a distinctive inherent pattern. However, in contrast to the more strongly sorbed odorants, the responses to this weakly sorbed odorant displayed little gradient in response magnitude along the 


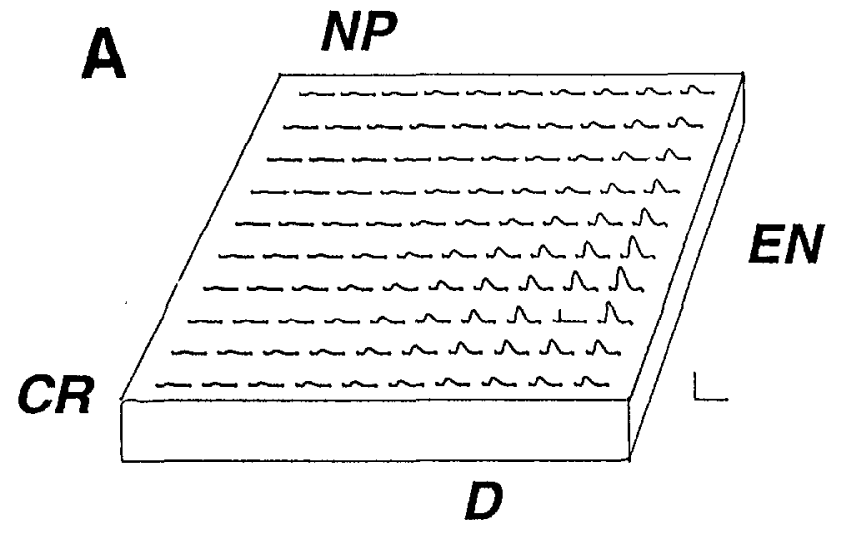

B
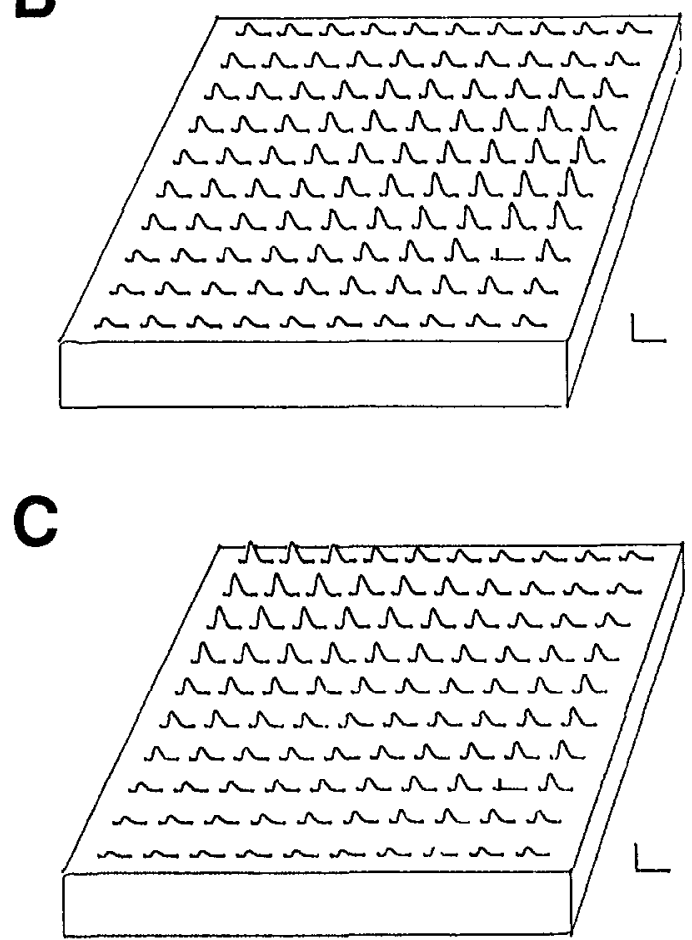

Figure 3. Fluorescent responses (di-4-ANEPPS) recorded from a $10 \times 10$ photodiode array matrix of the mucosa lining the medial surface of the turbinates. The odorant was carvone at $30 \%$ of vapor saturation. Horizontal lines, $32.25 \mathrm{sec}$; vertical lines, $1.0 \%$ change in fluorescence. One pixel (bottom right) is used to display a stimulus marker. $A$, Carvone drawn along the mucosa at a low flow rate $110 \mathrm{cc} / \mathrm{min} ; B$, carvone drawn along at high flow rate at $440 \mathrm{cc} / \mathrm{min} ; C$, carvone puffed down at $220 \mathrm{cc} / \mathrm{min} . E N$, external naris; $N P$, nasopharynx; $D$, dorsal; $C R$, cribriform platc.

flow path at the low flow rate and little difference between the inherent and the composite patterns regardless of flow rate. Furthermore, as flow rate was increased, the pixel-by-pixel response magnitudes, in sharp contrast to those of the strongly sorbed odorants, decreased with the increasing flow rate.

The effect of flow rate on response magnitude for each of the

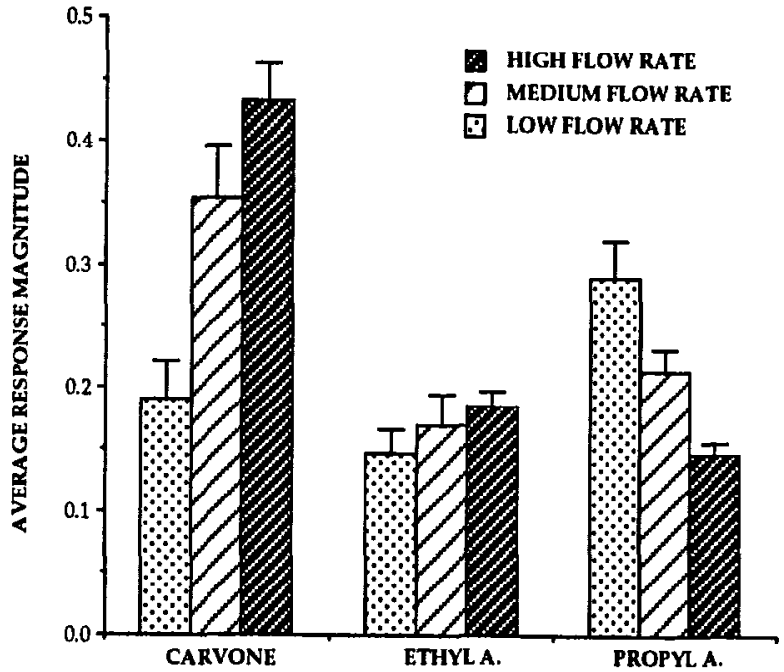

Figure 4. Effect of flow rate on response magnitude. SE bars are shown. Response magnitude is in pcrecntage change of fluorescence. Sce Results for further details.

three odorants at each of the three flow rates across all eight rats is shown quantitatively in Figure 4. To generate this figure, the average response magnitude across all 100 pixels was calculated first for each of the 100 pixels given by a particular odorant at a particular flow rate for a particular animal. These average response magnitudes for each odorant at each flow rate were then averaged across all eight animals. It is clear from Figure 4 that what was seen in Figure 3 for one animal is true across animals. That is, for the strongly sorbed odorants, carvone and ethyl acetoacetate, there was an increase in response magnitude with increasing flow rate. This effect was much more marked for carvone than for ethyl acetoacetate, which follows from the former's greater sorption strength. On the other hand, for propyl acetate, which is sorbed only weakly to the mucosa, increasing the flow rate gave a decided decrease in response magnitude. Thesc results are consistent with previous findings and explanations (Mozell et al., 1991). That is, these authors suggest that as flow rate increases, the dwell time of a sample of odorant molecules at any point above the mucosa is decreased so that for weakly sorbed odorants the response simply decreases. However, this effect is offset for strongly sorbed odorants because increasing the flow rate decreases previous absorption earlier in the flow path, allowing a greater number of molecules to reach the downstream sites.

To quantify and thereby facilitate the further comparison of the activity patterns for different odorants at different flow rates across all eight animals, the response in each of the 100 pixels for each set of conditions was "equilibrated" in relation to the entire 100 pixel array. That is, all 100 pixels in an array were "equilibrated" by dividing each of them by the average of all of them. This equilibration removed any differences in the absolute magnitudes of the responses within and among arrays, thereby keeping differences in absolute magnitude from being confused with

Figure 5. Color-enhanced surface plots for the rat's composite, inherent, and imposed activity patterns on the medial surface of the turbinates in response to carvone, ethyl acetoacetate, and propyl acetate when drawn through at either $440 \mathrm{cc} / \mathrm{min}$ (top) or $110 \mathrm{cc} / \mathrm{min}$ (bottom). The z-axis, which shows the relative response magnitudes, corresponds to the ratio of equilibrated responses for a given odorant compared with the standard amyl acetate response See Results for details. The natural logarithm of the ratios varied from -1.0 to 0.5 as indicated by the color bar to the right. $E N$, external naris; $N P$, nasopharynx; $D$, dorsal; $C R$, cribriform plate. 


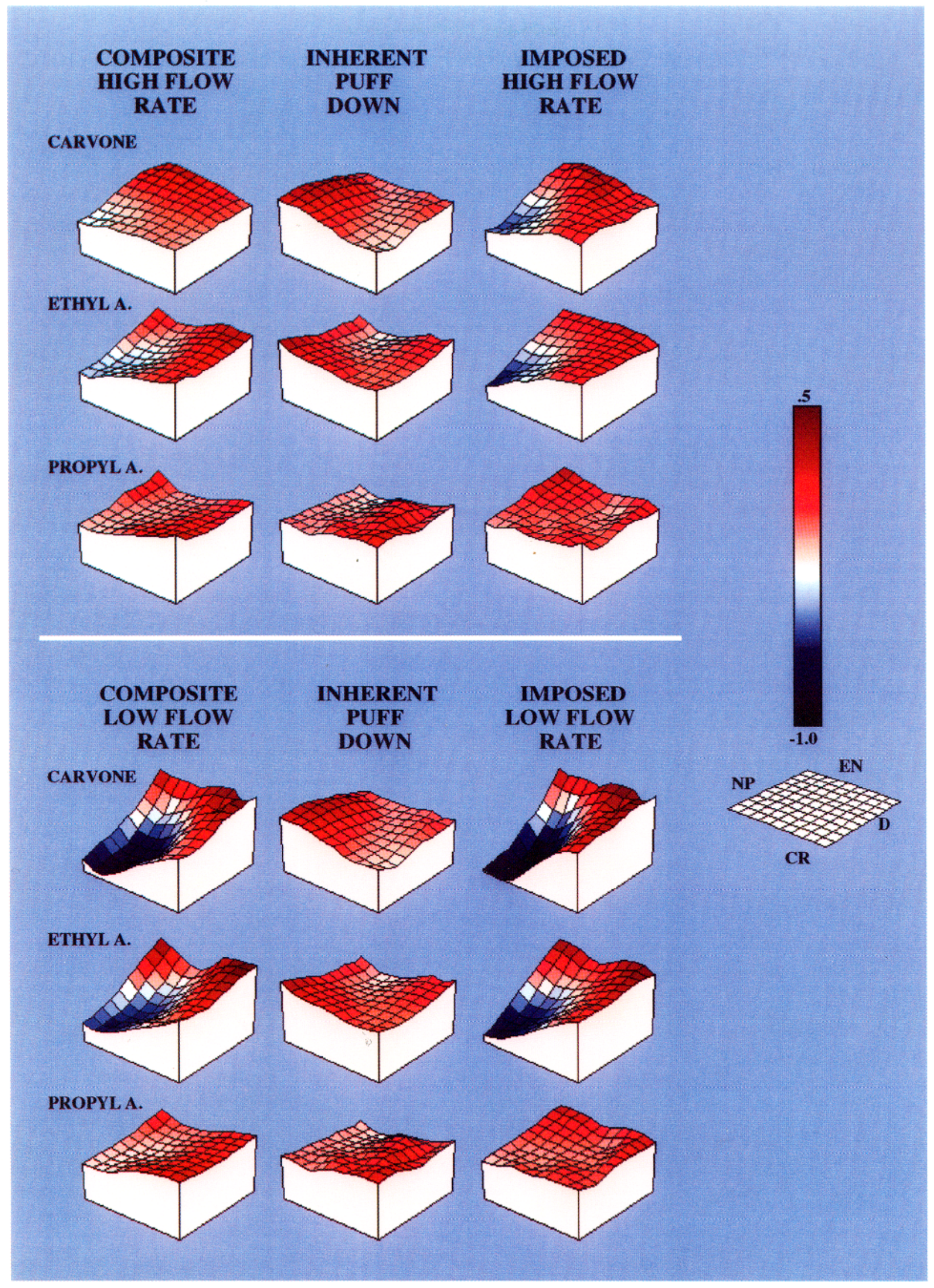


the differences in relative magnitude that define the differential imposed, inherent, and composite activity patterns (Kent and Mozell, 1992). To highlight further the activity patterns given by each odorant independent of any spatial differences in responsivity built in across the whole mucosa (such as variations in the total density of cells) that would affect the response to all odorants, an additional mathematical strategy was used. First, all of the data were transformed to natural logarithms, and then the response array produced by the standard odorant, amyl acetate, under the corresponding stimulus conditions was subtracted pixel-by-pixel from each response array produced by each experimental odorant. This strategy was used successfully in previous work (Mozell et al., 1984) and conforms with the concept that the mechanisms basic to the inherent and imposed activity patterns likely interact in a multiplicative manner.

With the data treated as discussed above, Figure 5 gives the composite activity patterns (odorants drawn along the mucosa), the inherent activity patterns (odorants puffed down), and the imposed activity patterns for the three odorants averaged across the eight animals. In Figure 5, the composite and imposed patterns are shown at the high flow rate (top) and at the low flow rate (bottom). Note that in accordance with the protocol of this study, the same arrays for the inherent patterns are shown for all three odorants in both panels. As seen in Figure 5 (top), each odorant gave a differential composite and inherent activity pattern. In this regard, the carvone and ethyl acetoacetate inherent patterns showed the largest relative responses toward the nasopharynx but differed from each other in the latter's showing a relatively greater response along the dorsal edge and cribriform plate. Propyl acetate differed from the other two, with its largest response toward the dorsal edge.

As far as the composite patterns at the high flow rate are concerned (Fig. 5, top), carvone gave, unlike its inherent pattern, the greatest response toward the external nares. Ethyl acetoacetate, differing somewhat less from its inherent pattern, showed its greatest response along the dorsal edge. Propyl acetate, differing minimally from its inherent pattern, again showed its greatest response along the dorsal edge.

To derive the imposed patterns, the inherent patterns expressed in logarithms were subtracted, as introduced above, pixel-by-pixel from the logged composite patterns, and these arrays are shown for the three odorants at the far right column. The highly sorbed odorants, carvone and ethyl acetoacetate, showed, as expected from chromatographic principles, pronounced gradients along the flow path giving the smallest response at the nasopharynx. A case can be made that the weakly sorbed odorant, propyl acetate, also showed a small gradient toward the nasopharynx but, compared with the strongly sorbed odorants, the gradient was muted.

At the low flow rate (Fig. 5, bottom), different composite activity patterns again were seen for the different odorants, although the composite patterns for the two most strongly sorbed odorants, carvone and ethyl acetoacetate, were rather similar in showing very steep gradients along the flow path decreasing toward the nasopharynx. In contrast, for the composite activity pattern of the weakly sorbed odorant, propyl acetate, there was only a very gradual gradient in the same direction. In deriving the imposed patterns from the composite and inherent patterns, as described above, the gradients of activity for the strongly sorbed odorants again became even steeper (Fig. 5, bottom).

In comparing the composite and imposed patterns for the high and low flow rates (Fig. 5, top vs bottom), several points consistent with chromatographic principles emerge. First, the greater the

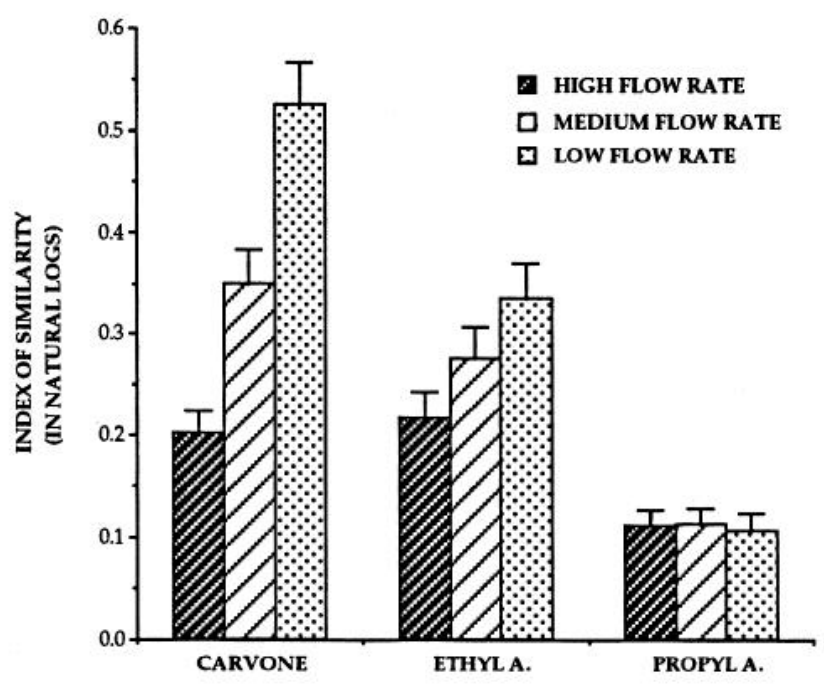

Figure 6. Composite pattern's change from inherent pattern as a function of flow rate. See Results for details.

flow rate the less pronounced the gradient along the flow path became, and this was demonstrated especially by the more strongly sorbed odorants. Second, as the gradient of the imposed pattern became steeper with decreasing flow rate, its contribution to the composite pattern appeared to increase. That is, especially for the more strongly sorbed odorants, the composite patterns came to resemble the imposed patterns more closely as flow rates decreased. This same effect could also be stated in the reverse, i.e., as flow rate was increased the inherent patterns became more dominant in the appearance of the composite patterns. In contrast, for the weakly sorbed odorant, propyl acetate, the imposed gradient was always small and the inherent pattern always dominated.

The decreasing influence of the mechanisms underlying the imposed patterns in generating the composite patterns as flow rate was increased is represented quantitatively in Figure 6. This figure uses a ratio that mathematically compares, for each odorant at each flow rate, the similarity between the activity pattern developed when the odorant was drawn along the mucosa (composite pattern) and that developed when the odorant was puffed down onto the mucosa (inherent pattern). That is, for each set of conditions, 100 ratios (one for each pixel) were expressed. The numerator in each ratio was the response magnitude, averaged over the eight animals, for a given pixel in the composite pattern array; the denominator was the averaged response magnitude for the corresponding pixel in the inherent pattern array. By taking the natural $\log$ of each of these ratios and adding their absolute values together, a logarithmic index of the similarity between the composite and inherent patterns was generated. The closer this index is to zero, the greater the similarity between the two patterns. Therefore, Figure 6 quantitatively establishes that the composite patterns differed from the inherent patterns, but the more the flow rate was increased the more the composite patterns came to resemble the inherent patterns. It further quantitatively documents that these effects were much more pronounced for the strongly sorbed odorants, carvone and ethyl acetoacetate, than for the weakly sorbed odorant, propyl acetate. Actually, for this weakly sorbed odorant the composite activity patterns differed only slightly from the inherent patterns regardless of the flow rate. 


\section{DISCUSSION}

Most of the reported work done at the mucosal level concerning the differential activity patterns produced by different odorants relates to the spatial aggregation of selectively sensitive receptors, i.e., the inherent activity patterns. However, these studies, with few exceptions, puff the odorant down onto the mucosa. This puffdown presentation bypasses any effect that drawing the odorant along the mucosa, as is the natural mode, may have in the differential access of the molecules of different odorants to different regions having odorant-selective sensitivities.

The current investigation studied the impact on the resulting activity patterns by drawing an odorant along the mucosa as opposed to puffing it down. The puff-down, inherent patterns were similar to those reported previously in the rat (Youngentob and Kent, 1995; Youngentob et al., 1995). However, the imposed patterns had not been demonstrated previously in mammals, and they were indeed robust. These imposed patterns were consistent with the results and the chromatographic explanation reported earlier, as listed above, in work with amphibia using techniques other than voltage-sensitive dyes. Namely, the imposed patterns showed a falling gradient of activity along the flow path that became steeper as the sorptive strengths of the odorants increased and/or odorant flow rate decreased. In addition, as seen earlier with summated multiunit recordings (Mozell et al., 1991, 1992), an increase in flow rate gave an increase in response magnitude for strongly sorbed odorants but a decrease for weakly sorbed odorants (see Results for explanation). These consistencies between the imposed patterns in this study and those previously studied using other techniques argue against any concern that the voltage-sensitive dye application procedures could alter the mucosa's sorption and, thus, the imposed patterns.

As far as the impact of the imposed patterns on the inherent patterns is concerned, it is clear from the results of this study that drawing odorants along the mucosa can have a pronounced effect on the mucosal activity patterns. This effect, as might be expected from the chromatographic principles noted above, becomes more pronounced as the sorptive strengths of the odorants increase but less pronounced with increasing flow rate. That is, the relative contribution of the imposed and inherent patterns to the overall composite pattern depends on both the odorant's sorptive strength and the flow rate at which the odorant is drawn along the mucosa. For example, the composite pattern for the highly sorbed odorants, ethyl acetoacetate and carvone, especially at lower flow rates, is dominated by the imposed pattern. However, the composite pattern for the weakly sorbed odorant, propyl acetate, is dominated more by its inherent pattern.

Similar conclusions regarding the lability of these composite patterns with flow rate in rats can be interpreted from the work by Ezeh et al. (1995). These investigators demonstrated with EOGs that when an odorant is drawn through the intact rat nasal cavity, the activity patterns for the more strongly sorbed odorants (anisole and ethyl butyrate) became more muted with increasing flow rate, whereas relatively little change was observed for the more poorly sorbed odorants (hexane and limonene).

It should be noted that the current study again brings into use the term composite patterns, previously introduced (Mozell et al., 1987) in naming the types of odorant selective activity patterns recorded from the mucosa. In judging the need and appropriateness of this term, it should be taken into consideration that because odorants normally pass through the olfactory passageways, they are likely to produce neither pure inherent patterns nor pure imposed patterns, but rather some result of their interaction, namely, composite patterns.

If, as is often assumed, mucosal activity patterns are basic to olfactory discrimination, the results of the present study raise questions about how the olfactory process deals with the lability of these patterns. There are a number of strategies that could be invoked in the olfactory process to regain confidence in mucosal patterns as encoding mechanisms. Some strategies, such as having the animal sniff at high flow rate, simply might negate the chromatographic process. This study and previous studies (Mozell, 1970; Mozcll et al., 1987) have shown that incrcasing flow ratc blunts the chromatographic effect and, thus, may highlight the inherent pattern. Another possible strategy is that the animal always takes a distinctive sniff with a similar flow profile, thereby maintaining the integrity of the composite pattern. [That flow rate may play a critical role in encoding of odorants is given some credibility from work with laryngectomized patients in whom increased sniff vigor increases olfactory discrimination (Schwartz et al., 1987).] Finally, processing at higher levels may obviate imposed patterns. For example, the system may attend only to those glomeruli that are excited and not to the relative gradation of their responses.

Other strategies, instead of negating, actually might take advantage of the chromatographic process to achieve additional discriminative permutations. Two such scenarios unfold in Figure 7. This figure indicates the direction of the odorant's movement across the mucosa-lined turbinates by plotting, as equal time contours (isochrones), the response times averaged across all eight animals to the low flow rate of propyl acetate. Similar isochrone maps were obtained for the medium and high flow rates, except that the times, as expected, were proportionally shorter. In the first scenario, these spatially distributed temporal patterns themselves may play a role in the encoding of odorants at the level of the olfactory bulb. That is, those mucosal regions that activate most rapidly may alter, through lateral inhibition, the spatial bulbar patterns of glomeruli activity. The second scenario begins by noting that the odorant-laden air may be inferred from Figure 7 to flow perpendicularly to the isochrone lines. If, as done in Figure 7, the three bands of different receptor types reported by Vassar et al. (1993) are added, it becomes clear that these bands are also perpendicular to the isochrones, i.e., they are parallel to the odorant flow. Therefore, if as Vassar et al. (1993) suggest, cach band contains a particular family of rcceptors homogeneously distributed throughout the band, the mucosa could do a dual encoding of the flowing odorants: (1) determine which combination of bands is responding, i.e., the inherent patterns; and (2) determine how far along each band the response extends, i.e., the imposed patterns. Take, as a purely hypothetical example, a homologous series of alcohols, all members of which with their similar chemical properties might excite the same combination of bands. However, as their chain lengths vary, and thereby their physicochemical sorption properties, the molecules of different members of the series might reach and excite different extents of the bands, thus producing different composite patterns.

The system also could take advantage of the chromatographic process by separating the odorants in a mixture in space and time along the flow path before the olfactory mucosa. 'I his separation could be enhanced further by the temporal differences in mucosal activation between different odorants. Future experiments will determine whether such scenarios are actually tenable.

Recent anatomical work that detects odorant receptor mRNA in the axons of olfactory neurons has shown that 
Figure 7. Isochrone map of start times (solid lines) for propyl acetate at $110 \mathrm{cc} / \mathrm{min}$ superimposed on the olfactory mucosa lining the medial surface of the turbinates. The three bands of different receptor types reported by Vasser et al. (1993) also are superimposed (stippling and slanted lines) on the olfactory mucosa and appear to be perpendicular to the isochrone lines (i.e., parallel to flow of odorant laden air). $E N$, external naris; $N P$, nasopharynx; $D$, dorsal; $C R$, cribriform plate. (This map is corrected for the very small variations in the start times of the inherent patterns across the mucosa when the odorant is puffed down. This correction is identical to that described in Results for obtaining the imposed activity patterns from the composite patterns, except that timing rather than response magnitude is used. As in the patterns based on response magnitude, the start time differences of the inherent patterns were much smaller than those of the imposed patterns.)

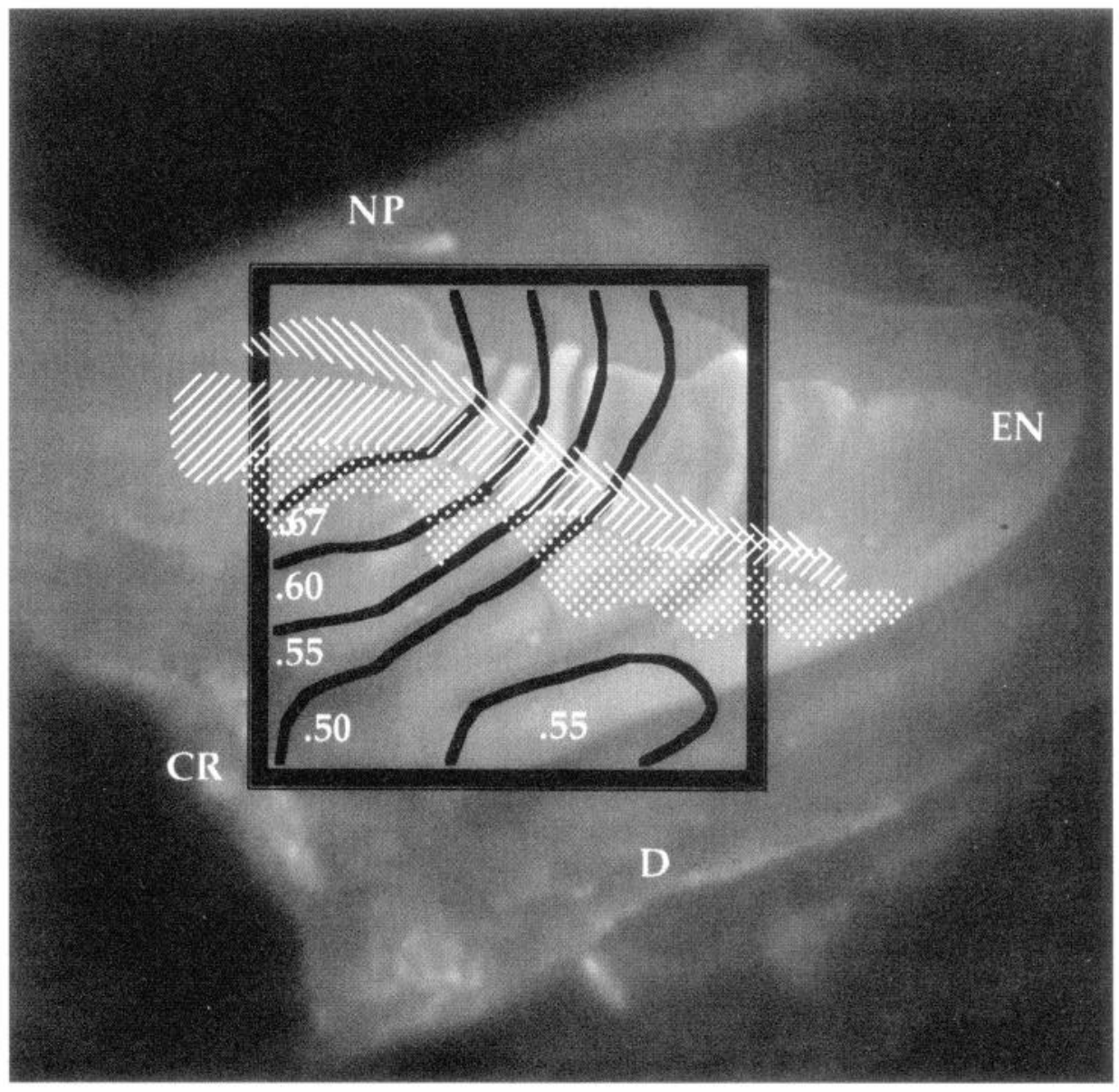

specific odorant receptor gene probes converge onto a very small number of glomeruli that are organized spatially on the olfactory bulb in a stereotypical manner from animal to animal (Ressler et al., 1994; Vassar et al., 1994). Further, the number of proposed odorant receptor genes across species seems to be consistent with the number of glomeruli present (Vassar et al., 1994). These observations suggest that the quality of an odorant can be represented in the pattern of glomeruli that respond uniquely to a given odorant. The hypothesis that this putative code is represented on the mucosa is supported by the observation that the odorant receptors are aggregated spatially in large, homogeneous, longitudinal zones (Vassar et al., 1993; Ressler et al., 1994) and clusters (Strotmann et al., 1994). These zones and clusters, which are consistent in position across animals, display symmetry from the right and left nasal cavities and on the medial surfaces of the septum and turbinate. In the current study, all odorants elicited responses at all positions on the epithelium, although at different relative magnitudes, implying that a given odorant interacts with many different receptor types. The puff-down, inherent patterns of odorant-elicited activity resemble a clustering more than a zonal pattern. This may reflect a number of possibilities: (1) the odorants chosen; (2) an inadequate resolution by the voltagesensitive dye-recording technique; or (3) the differential access of odorants to receptors at different mucosal locations even during the puff-down procedure.

In the meantime, it is clear that the differential activity patterns induced by different odorants across the olfactory mucosa are indeed labile to the stimulation conditions. This lability must be considered henceforth when assessing the putative role of mucosal activity patterns in the coding of odorants.

\section{REFERENCES}

Cinelli AR, Hamilton KA, Kauer JS (1995) Salamander olfactory bulb neuronal activity observed by video rate, voltage-sensitive dye imaging. III. Spatial and temporal properties of responses evoked by odorant stimulation. J Neurophysiol 73:2053-2071.

Ezeh PI, Davis LM, Scott JW (1995) Regional distribution of rat electroolfactogram. J Neurophysiol 73:2207-2220.

Hornung DE, Mozell MM (1977) Factors influencing the differential sorption of odorant molecules across the olfactory mucosa. J Gen Physiol 69:343-361.

Hornung DE, Mozell MM (1981) Accessibility of odorant molecules to the receptors. In: Biochemistry of olfaction and taste (Cagan R, Kare M, eds), pp 33-45. New York: Academic.

Hornung DE, Mozell MM, Serio JA (1980) Olfactory mucosa/air partitioning of odorants. In: Olfaction and taste, Vol VII (Van der Starre H, ed), pp 167-170. London: IRL.

Kauer JS, Moulton DG (1974) Response to olfactory bulb neurons to odor stimulation of small nasal areas in the salamander. J Physiol (Lond) 243:717-737.

Kent PF, Mozell MM (1992) The recording of odorant-induced mucosal activity patterns with a voltage-sensitive dye. J Neurophysiol 68:1804-1819.

Kubie JL, MacKay-Sim A, Moulton DG (1980) Inherent spatial patterning of response to odorants in the salamander olfactory epithelium. In: Olfaction and taste, Vol VII (Van der Starre H, ed), pp 163-166. London: IRL.

MacKay-Sim A, Kesteven S (1994) Topographic patterns of responsiveness to odorants in the rat olfactory epithelium. J Neurophysiol $71: 150-160$ 
MacKay-Sim A, Kubie JL (1981) The salamander nose: a model system for the study of spatial coding of olfactory quality. Chem Scnses $6: 249-257$.

MacKay-Sim A, Shaman P, Moulton DG (1982) Topographic coding of olfactory quality: odorant specific patterns of epithelial responsivity in the salamander. J Neurophysiol 48:584-596.

Moulton DG (1976) Spatial patterning response to odors in the peripheral olfactory system. Physiol Rev 56:578-593.

Mozell MM (1964) Evidence for sorption as a mechanism of the olfactory analysis of odorants. Nature 203:1181-1182.

Mozell MM (1966) The spatiotemporal analysis of odorants at the level of the olfactory receptor sheet. J Gen Physiol 50:25-41.

Mozell MM (1970) Evidence for a chromatographic model of olfaction. J Gen Physiol 56:46-63.

Mozell MM, Jagodowicz M (1973) Chromatographic separation of odorants by the nose: retention times measured across in vivo olfactory mucosa. Science 181:1247-1249.

Mozell MM, Jagodowicz M (1974) Mechanisms underlying the analysis of odorant quality at the level of the olfactory mucosa. I. Spatiotemporal sorption patterns. Ann NY Acad Sci 237:76-90.

Mozell MM, Kent PF, Murphy SJ (1991) The effect of flow rate on the magnitude of the olfactory response differs for different odorants. Chem Senses 16:631-649.

Mozell MM, Kent PF, Murphy SJ (1992) An addendum to the effect of flow rate on the olfactory response: the role of odorant dwell time in the olfactory sac. Chem Senses 17:583-587.

Mozell MM, Sheehe PF, Hornung DE, Kent PF, Youngentob SL, Murphy SJ (1987) "Imposed" and "inherent" mucosal activity patterns: their composite representation of olfactory stimuli. $\mathfrak{J}$ Gen Physiol $90: 625-650$.
Mozell MM, Sheehe PR, Swieck SW, Kurtz DB, Hornung DE (1984) A parametric study of the stimulation variables affecting the magnitude of the olfactory nerve response. J Gen Physiol 83:233-267.

Ressler KJ, Sullivan SS, Buck LB (1994) Information coding in the olfactory system: evidence for a stereotyped and highly organized epitope map in the olfactory bulb. Cell 79:1245-1255.

Schwartz DN, Mozell MM, Youngentob SL, Leopold DL, Sheehe PR (1987) Improvement of olfaction in laryngectomized patients with the larynx bypass. Laryngoscope 97:1280-1286.

Strotmann J, Wanner I, Helfrich T, Beck A, Breer H (1994) Rostrocaudal patterning of receptor-expressing olfactory neurones in the rat nasal cavity. Cell Tissue Res 278:11-20.

Thommesen G, D'Oving KB (1977) Spatial distribution of the EOG in the rat: a variation with odor quality. Acta Physiol Scand 99:270-280.

Vassar R, Chao SK, Sitcheran R, Nunez JM, Vosshall LB, Axel R (1994) Topographic organization of sensory projections to the olfactory bulb. Cell 79:981-991.

Vassar R, Ngai J, Axel R (1993) Spatial segregation of odorant receptor expression in the mammalian olfactory epithelium. Cell 74:309-318.

Youngentob SL, Kent PF (1995) Enhancement of odorant-induced mucosal activity patterns in rats trained on an odorant identification task. Brain Res 670:82-88.

Youngentob SL, Kent PF, Sheehe PR, Schwob JE, Tzoumaka E (1995) Mucosal inherent activity patterns in the rat: evidence from voltagesensitive dyes. J Neurophysiol 73:387-398.

Youngentob SL, Markert LM, Hill TW, Matyas EP, Mozell MM (1991) Odorant identification in rats: an update. Physiol Behav 49:1293 -1296.

Youngentob SL, Mozell MM, Sheehe PR, Hornung DE (1987) A quantitative analysis of sniffing strategies in rats performing odor detection tasks. Physiol Behav 41:59-69. 